\title{
Timing of Incident STI Relative to Sex Partner Change in Young Women
}

\author{
Mary A. Ott, MD, MA ${ }^{\mathrm{a}}$, Jaroslaw Harezlak, $\mathrm{PhD}^{\mathrm{b}}$, Susan Ofner, $\mathbf{M S}^{\mathrm{b}}$, and J. Dennis \\ Fortenberry, MD, MS \\ aSection of Adolescent Medicine, Department of Pediatrics, Indiana University School of \\ Medicine, Indianapolis, IN, USA \\ ${ }^{b}$ Department of Biostatistics, Indiana University School of Medicine, Indianapolis, IN, USA
}

\section{Abstract}

Among adolescents, partner changes are associated with STIs, but little is known about timing. Using daily diaries and weekly STI tests, we describe whether infections occur before or after partner change during periods when young woman changes partners once. Results showed infections occurring both before and after partner changes.

\section{Keywords}

Adolescent; Sexual Behavior; Concurrency; Sexual Relationships; Sexually Transmitted Infection; Gonorrhea; Chlamydia; Trichomonas; incidence; Female

Adolescent females have the highest age-specific rates of gonorrhea and chlamydia. ${ }^{1,2}$ Acquisition of these bacterial STI has been linked to partner change, 3,4 including, "serial monogamy", or sequential non-overlapping partners. ${ }^{3}$ Little is known about the timing of STI transmission within partner changes, yet this information can inform STI prevention. In serial monogamy, in theory, STI transmission occurs as long as the gap between partners is shorter than the infectious period of the organism. ${ }^{5}$ In reality, many adolescents are unaware about their partner's infidelity, ${ }^{6}$ and the detailed sexual network information needed to be assured of monogamy by both sex partners is rarely available to clinicians or researchers. Partner change is frequently a time of relationship turmoil. ${ }^{7,8}$ If a partner change is a reflection of partner distrust or suspicion that a partner is unfaithful, then STI may precede the partner change.

An analysis of where infection occurs with respect to partner change requires frequent assessments of sexual behavior and STIs. Using a unique data set that included daily diaries of sexual behavior and weekly STI screening tests, we conducted an exploratory analysis of whether gonorrhea, chlamydia and trichomonas infections are acquired before or after partner change among young women reporting serial monogamy, or one partner change with no overlap.

As part of a larger IRB approved study of STI, 14-17 year old females were recruited from three adolescent clinics serving communities with high STI prevalence, and followed for up to 8 years. Each year, participants provided two 3-month periods of daily diaries of partner-

Contact Information: Mary A. Ott, MD MA, Associate Professor of Pediatrics, Section of Adolescent Medicine, Indiana University School of Medicine, 410 West $10^{\text {th }}$ Street, HS 1001, Indianapolis, IN 46202, (317) 274-8812, (317) 274-0133 fax, maott@iupui.edu. 
specific sexual behaviors, as well as weekly self-collected vaginal swabs for chlamydia, gonorrhea and trichomonas amplified DNA-based testing. Positive gonorrhea tests were verified by a different DNA-based test. Tests were batched, and done at the end of the 3month period, at which point participants were informed and treated per Centers for Disease Control and Prevention guidelines. ${ }^{9}$ Details of the larger study are provided elsewhere. ${ }^{10}$

The units of analysis were any given 3-month-diary-periods over the course of the study that met the following conditions. First, participants reported sex partner change during the diary period ( $\mathrm{n}=274$ ). (In most periods, participants reported no partners or 1 partner). Second, to identify whether the infection occurred before or after partner change, we limited our analysis to intervals in which participants reported only two sex partners $(\mathrm{n}=205)$, and did not switch back-and-forth between partners $(\mathrm{n}=141)$. Third, to focus on incident STI, we eliminated diary periods in which the first swab of the period was positive for STI, leaving 111 intervals for analysis. Finally, when multiple periods from the same participant were used, periods were at least 5 months apart to minimize within-subject dependence.

Because of organism differences in transmission, Chlamydia, trichomonas, and gonorrhea were analyzed separately. The date of partner change was the first report of sex with the second partner. The date of STI acquisition was the first weekly swab to test positive for that STI. STIs occurring before the date of partner change were classified as interval 1; STIs occurring on or after the date of partner change, interval 2.

In the context of partner change, it is assumed that incidence of infections with the second partner (interval 2) will be greater than with the first partner (interval 1). Therefore, our null hypothesis was that the proportion of infections in interval $2\left(\mathrm{p}_{2}\right)$ would be greater than that of interval $1\left(\mathrm{p}_{1}\right)$, by some constant (c) amount:

$$
\mathrm{H}_{0}: \quad \mathrm{p}_{2}>\mathrm{p}_{1}+\mathrm{c}
$$

To estimate the differences in the probability of infection in interval 1 versus interval 2, we used a non-inferiority type of two-sample test with a one-sided $95 \%$ confidence interval. A logistic regression model was used to estimate the odds ratios of STI in interval 1 vs. interval 2 . We provide the $95 \%$ confidence intervals as well as non-inferiority test results showing the smallest detectable odds ratio for a significance level alpha equal to 0.05 . We interpret the upper bound of the $95 \%$ CI to mean that, if a difference exists between proportion of STIs diagnosed before and after partner change, there is only a 5\% chance that the difference will be larger than the upper limit of the 95\% CI.

Ninety-two participants provided 111 diary periods. Most (83\%) contributed one period, 14 contributed two, and one each three and four periods. Participants were $94 \%$ African American. The mean age of participants at the time the data were collected was 17.8 years.

Figure 1 shows results for timing of sex and infection for six representative periods. We observed different patterns of positive tests in interval 1 versus interval 2 for chlamydia, gonorrhea and trichomonas. For chlamydia infections, incident infections occurred both before and after partner change, with $11(9.9 \%)$ of diary periods positive in interval 1, and $10(9.0 \%)$ positive in interval 2 . Similarly for trichomonas, infections were observed both in interval 1, with $8(7.2 \%)$ of diary periods positive in interval 1, and $5(4.5 \%)$ positive in interval 2. For gonorrhea, more infections were observed after partner change, in interval 2, with $3(2.7 \%)$ of diary periods positive in interval 1 , and $9(8.1 \%)$ positive in interval 2.

For chlamydia and trichomonas, we detected a moderate difference in incidence between the two intervals (see table 1). For these two organisms, we had adequate power at $\mathrm{p}<.05$ to 
detect an odds ratio of 1.8 or higher for not detecting an infection in interval 2 versus 1. Restated, if there is a difference in the odds of acquiring an infection in interval 2 versus 1 , we are $95 \%$ certain it is not greater by more than 1.8 . In contrast, for gonorrhea, the smallest detectable OR had an upper bound of 10 . This means that, if there is a difference between interval 2 and interval 1, we are $95 \%$ certain it is not greater by more than 10 . This high upper bound indicates that, for gonorrhea, interval 2 may have a much higher rate than interval 1.

This analysis extends our understanding of the association between partner change and STI acquisition. Despite the limitations of a small sample, we were able to demonstrate that many infections occur before partner change, suggesting that, in these cases, the infection was acquired from the first partner, rather than the second partner. In these cases, the STI risk was in the first relationship "going bad," rather than the partner change itself. This is complementary to network analyses, which can miss as many as 40-60\% of sexual partnerships. ${ }^{11}$ Our use of an urban African American sample from a community with high rates of STIs may limit transferability of results to other populations; However, it is a group of importance to study because of marked STI-related health disparities.

Also of importance are organism-specific patterns of infection. This may be due in part to differences in transmissibility. Gonorrhea has been shown to be highly transmissible, and Chlamydia less so. ${ }^{12}$ These patterns may also reflect differences in the development of symptoms, assuming that individuals with symptoms might have sought care and received an STI diagnosis and treatment.

These findings are consistent with research showing that young women are frequently unaware of their partners' other partners, ${ }^{6}$ and suggest that clinicians may want to more carefully inquire about relationships, and consider enhanced screening in young women reporting relationships of lower quality, with lower levels of communication, or in turmoil.

\section{Acknowledgments}

Sources of Support: NIH K23-HD049444-01, R01 HD 044387

\section{References}

1. Gavin L, MacKay AP, Brown K, et al. Sexual and reproductive health of persons aged 10-24 years United States, 2002-2007. MMWR Surveill Summ. Jul 17; 2009 58(6):1-58. [PubMed: 19609250]

2. Centers for Disease Control and Prevention. Sexually Transmitted Disease Surveillance, 2010. Atlanta, GA: U.S. Department of Health and Human Services; 2011.

3. Kelley SS, Borawski EA, Flocke SA, Keen KJ. The role of sequential and concurrent sexual relationships in the risk of sexually transmitted diseases among adolescents. J Adolesc Health. Apr; 2003 32(4):296-305. [PubMed: 12667734]

4. Niccolai LM, Ethier KA, Kershaw TS, Lewis JB, Meade CS, Ickovics JR. New sex partner acquisition and sexually transmitted disease risk among adolescent females. J Adolesc Health. Mar; 2004 34(3):216-223. [PubMed: 14967345]

5. Foxman B, Newman M, Percha B, Holmes KK, Aral SO. Measures of Sexual Partnerships: Lengths, Gaps, Overlaps, and Sexually Transmitted Infection. Sex Transm Dis. Apr; 2006 33(4):209-214. [PubMed: 16434884]

6. Lenoir CD, Adler NE, Borzekowski DLG, Tschann JM, Ellen JM. What you don't know can hurt you: Perceptions of sex-partner concurrency and partner-reported behavior. Journal of Adolescent Health. Mar; 2006 38(3):179-185. [PubMed: 16488813]

7. Ott MA, Katschke A, Tu W, Fortenberry JD. Partner Change, Relationship Characteristics and STI acquisition in Adolescent Women. Sexually Transmitted DIseases. 2011; 38(3):153-7. [PubMed: 20852455] 
8. Sayegh MA, Fortenberry JD, Anderson J, Orr DP. Relationship quality, coital frequency, and condom use as predictors of incident genital Chlamydia trachomatis infection among adolescent women. J Adolesc Health. Aug.2005 37(2):163. [PubMed: 16026726]

9. Centers for Disease Control and Prevention. Sexually Transmitted Disease Treatment Guidelines, 2006. Atlanta, GA: Department of Health and Human Services; 2006.

10. Fortenberry JD, Temkit M, Tu W, Graham CA, Katz BP, Orr DP. Daily mood, partner support, sexual interest, and sexual activity among adolescent women. Health Psychol. May; 2005 24(3): 252-257. [PubMed: 15898860]

11. Ghani AC, Donnelly CA, Garnett GP. Sampling biases and missing data in explorations of sexual partner networks for the spread of sexually transmitted diseases. Stat Med. Sep 30; 1998 17(18): 2079-2097. [PubMed: 9789915]

12. Lycke E, Lowhagen GB, Hallhagen G, Johannisson G, Ramstedt K. The risk of transmission of genital Chlamydia trachomatis infection is less than that of genital Neisseria gonorrhoeae infection. Sex Transm Dis. Jan-Mar;1980 7(1):6-10. [PubMed: 6771879] 


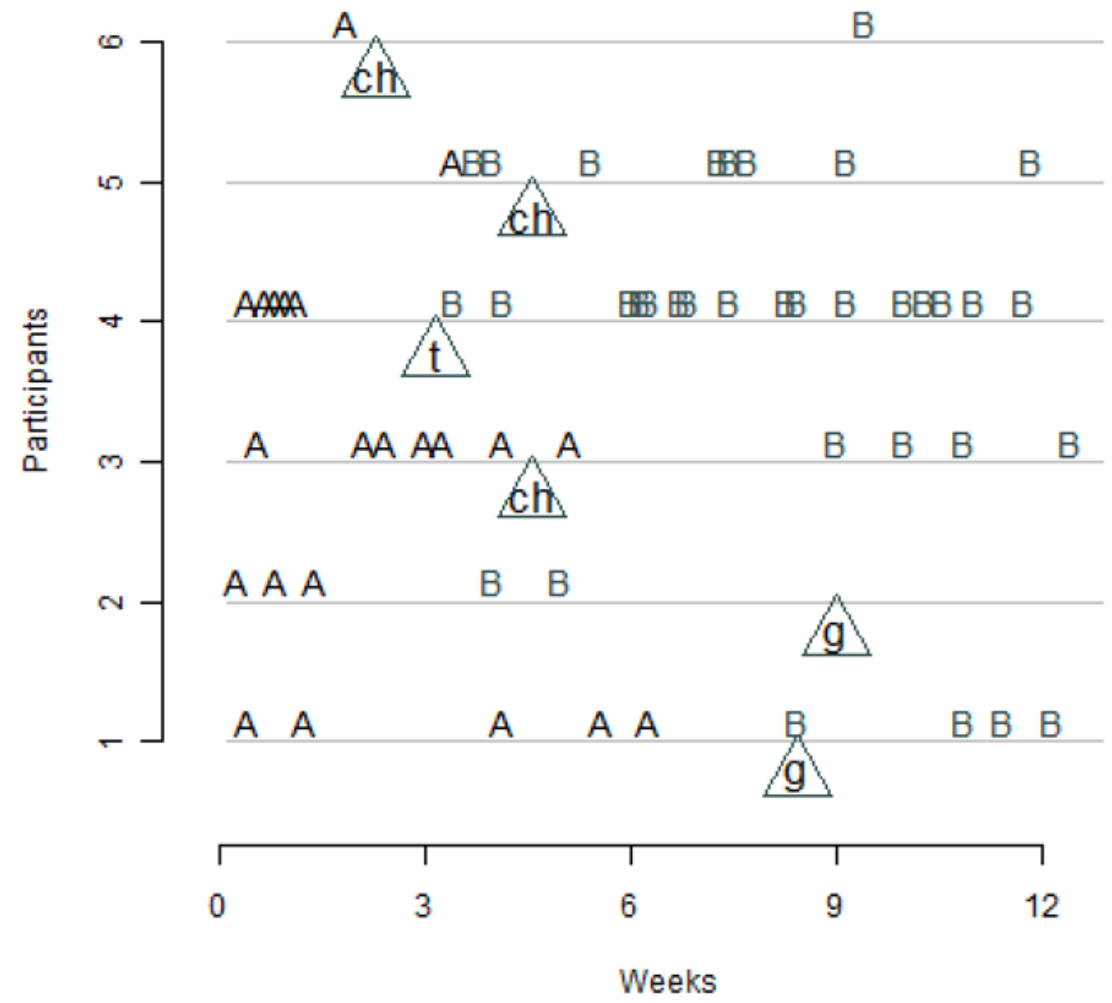

Figure 1.

Representative 12 Week Periods with Dates of Sex, Partner Change, and STI Diagnoses Key:

$A=$ Daily diary reports of sex with the first partner (Interval 1)

$\mathrm{B}=$ Daily diary reports of sex with the second partner (Interval 2)

$\mathrm{ch}=$ Date of $1^{\text {st }}$ weekly swab testing positive for chlamydia $\mathrm{g}=$ Date of $1^{\text {st }}$ weekly swab testing positive for gonorrhea $\mathrm{t}=$ Date of $1^{\text {st }}$ weekly swab testing positive for trichomonas 
TABLE 1

Number of Infections and Percentage in the Time Periods Corresponding to Runs 1 and 2

\begin{tabular}{llll}
\hline & Interval 1 Number (\%) & Interval 2 Number $(\%)$ & OR $(\mathbf{9 5 \%}$ CI) \\
\hline CT & $11(9.9 \%)$ & $10(9.0 \%)$ & $0-1.8$ \\
GC & $3(2.7 \%)$ & $9(8.1 \%)$ & $0-9.7$ \\
TV & $8(7.2 \%)$ & $5(4.5 \%)$ & $0-1.5$ \\
Any STI & $21(18.9 \%)$ & $19(17.1 \%)$ & $0-1.7$ \\
\hline
\end{tabular}

Number and proportion of STIs diagnosed before partner change (Interval 1) or after partner change (Interval 2). If a difference exists between proportion of STIs diagnosed before and after partner change, there is only a 5\% chance that the difference will be larger than the upper limit of the $95 \%$ CI. 